\title{
FURTHER STUDIES ON THE MOSQUITO FAUNA OF PUNJAB, INDIA
}

Jagbir S. Kirti and Jagdish Kaur

Department of Zoology, Punjabi University, Patiala, Punjab 147002, India

\section{ABstract}

A total of thirty species of mosquitoes have been collected and identified from Punjab. A list of these species alongwith their first reference, material examined and distribution is given in this manuscript. Three species have been reported as new records from this state.

\section{KEYWORDS \\ India, Mosquito, Punjab, checklist}

Negligible information has been added to the mosquito fauna of Punjab since the publication of the monographs on Indian anophelines by Christophers (1933) and culicines by Barraud (1934), except for two short communications by Sagandeep et al. (1994) and Kirti and Kaur (1999). Moreover, the outbreaks of dengue in Ludhiana District during 1996 and 1999 and Japanese encephalitis in Fatehgarh District warrant the study of mosquito fauna in the changed scenario of this agricultural state. The present communication is intended to place on record the information collected on mosquito fauna during intensive and extensive surveys in various districts of Punjab during 1997-2000.

\section{Material And Methods}

Immature as well as mature mosquito specimens were collected from cattlesheds, human dwellings, water pools, ponds, rivulets, tree holes and domestic containers. The immature stages were reared up to the adult emergence stage. Adult specimens were killed with ethyl acetate vapours. The freshly killed specimens were pinned and preserved in air tight insect cabinets. As many as 1,551 adult representatives of mosquitioes belonging to 30 species referable to six genera of subfamilies Anophelinae and Culicinae were collected and identified. The identification of various species was done with the help of relevant literature (Christopher, 1933; Barraud, 1934; Puri, 1954; Wattal \& Kalra, 1961).

\section{Results}

Genus Anopheles Meigen

Meigen, 1818, Syst. Beschr., 1: 10

Type species: Anopheles claviger Meigen

\section{Anopheles (Anopheles) paraliae Sandosham}

Material examined: Nine females, 8.iv.2000, Harike, Amritsar, coll. J. Kaur.

\section{Anopheles (Anopheles) peditaeniatus (Leicester)}

Material examined: 10 females, 2.v.1997, eight females, 25.ix.1997, Mohie, Ludhiana; 10 females, 3.ix.1997, 10 females, three males, 21.ix.1997, one female, three males, 8.xi.1998, Patiala, coll. J. Kaur.

3. Anopheles (Anopheles) nigerrimus Giles

Material examined: One male, 21.ix.1997, Patiala; one female 25.ix.1999, Mohie, Ludhiana, coll. J. Kaur.

\section{Subgenus Cellia Theobald}

Theobald, 1903, Monog. Culic., 3: 107

Type species: Anopheles pharoensis Theobald

\section{Anopheles (Cellia) stephensi Liston}

Material examined: Four females, 30.iv.1997, Patiala; two females, 5.vii.1997, Mullanpur, Ludhiana; seven females, 17 males, 21.vii.1997, Bangsipur; 37 females, 16 males, 6.vii.1998, Mohie, Ludhiana; four females, 29 males, 23.vii.1999, Handiaya, Barnala, coll. J. Kaur.

\section{Anopheles (Cellia) pulcherrimus Theobald}

Material examined: One female, 1.vii.1998, Patiala; three females 13.vii.1998, Mohie, Ludhiana; two females, 21.vii.1998, Bangsipur, coll. J. Kaur.

\section{Anopheles (Cellia) annularis Van der Wulp}

Material examined: 12 Females, 3.vii.1997, five females, one male, 8.vii.1998, Patiala; one female, 11.viii. 1998, Bela Dhiani, Nangal; seven females, 7.vii.1999, Rahon, Nawan Shahr, coll. J. Kaur.

\section{Anopheles (Anopheles) subpictus Grassi}

Material examined: One female, 11.vii.1998, Nangal, coll. J. Kaur.

\section{Anopheles (Cellia) culicifacies Giles}

Material examined: One female, 21.vii.1997, Bangsipur, Ludhiana; 31 females, 11.viii.1998, Nangal; 11 females 12.viii.1998, Anandpur Sahib, coll. J. Kaur.

\section{Anopheles (Cellia) fluviatilis James} Material examined: Three females, 11.viii.1998, Bela Dhiani, Nangal, coll. J. Kaur.

\section{Genus Mansonia Blanchard}

Blanchard, 1901, C.R. Soc. Biol., 53: 1045

Type species: Culex titillans Walker

\section{Mansonia (Mansonioides) uniformis (Theobald)}

Material examined: Five females, 1.x.1997, two females 22.ix.1999, Patiala; two females, 10.viii.1998, Anandpur Sahib; six females, 8.iv.2000, Harike, Amritsar, coll. J. Kaur and J. Singh.

11. Mansonia (Mansonioides) dives (Schiner, 1868). Material examined: One female, 29.iv.1999, two females, 8.iv.2000, Harike, Amritsar, coll. J. Kaur and J. Singh.

Genus Armigeres Theobald

Theobald, 1901, Monog. Culic., 1: 322.

Type species: Armigeres obturbans (Walker)

\section{Armigeres (Armigeres) subalbatus (Coquillett)}

Material examined: Two females, two males, 21.vi.1997, 12 females, 30 males, 6.viii.1997, 14 females, 1.x.1997, three females,10.xi.1997, 17 females, two males, 4.iv.1998, Patiala; two females, two male, 5.vii.1997, one female, 7.iv.1998, Mohie, Ludhiana; one female, two males, 1.iii.1998, Bassi Pathana, Fatehgarh Sahib; four females, 23.iv.1998, Mukeria, Hoshiarpur; one female, one male, 2.v.1998, Talwara, four females, 17.x.1998, Bhaiyan, Gurdaspur, three females, 12 males, 9.vii.1999, Hajipur; one female, one male, 8.v.1998, Anandpur Sahib, Ropar, coll. J. Kaur and J. Singh. 
Genus Aedes Meigen

Meigen, 1818, Syst. Beschr., 1: 13.

Type species: Aedes cinereus Meigen

\section{Subgenus Stegomyia Theobald}

Theobald, 1901, Monog. Culic., 1: 283.

Type species: Culex fasciatus Fabricius

13. Aedes (Stegomyia) aegypti (Linnaeus)

Material examined: 10 females, four males, 5.ix.1999, Ludhiana; one female, 30.ix.1999, Patiala, coll. J. Kaur and J. Singh.

\section{Aedes (Stegomyia) albopictus (Skuse)}

Material examined: Six females, 18.viii.1997, five females, 27.viii.1997, 27 females, 10 males, 2.iv.1998, 17 males, 22.vii.1998, Patiala; 11 females, two males, 27.viii.1998, Chatbir, coll. J. Kaur.

\section{Aedes (Stegomyia) gardnerii (Ludlow)}

Material examined: One female, 2.iv.1998, Patiala; one female, 27.viii.1998, Chattbir, coll. J. Kaur.

16. Aedes (Stegomyia) w-albus Theobald

Material examined: One female, 2.iv.1998, Patiala; one female, one male, 27.viii.1998, Chattbir, coll. J. Kaur.

17. Verrallina (Neomacleaya) indica (Theobald, 1907).

Material examined: Four females, 3.ix.1997, five females, 26.ix.1998,

Patiala, coll. J. Singh.

\section{Subgenus Mucidus Theobald}

Theobald, 1901, Monog. Culic., 1: 268.

Type species: Culex alternans Westwood

18. Ochlerotatus (Mucidus) scatophagoides (Theobald, 1901). Material examined: One female, 24.ix.1997, Patiala, coll. J. Kaur.

\section{Subgenus Neomelaniconion Newstead}

Newstead, 1907, Ann. trop. Med. Parasit., 1: 31

Type species: Culex luteolateralis Theobald.

19. Aedes (Neomelaniconion) lineatopennis (Ludlow) Material examined: Three females, one male, 21.ix.1997, Patiala, coll J. Kaur.

Subgenus Aedimorphus Theobald

Theobald, 1903, Monog. Culic., 3: 290.

Type species: Uranotaenia domestica Theobald

20. Aedes (Aedimorphus) jamesi (Edwards)

Material examined: One female, 8.x.1998, Patiala, coll. J. Kaur.

Genus Mimomyia Theobald

Theobald, 1903, Monog. Culic., 3: 304

Type species: Mimomyia splendens Theobald

21. Mimomyia (Mimomyia) chamberlaini (Ludlow)

Material examined: Two males, 30.iv.1998, Mukerian, Hoshiarpur, coll.

J. Kaur.

Genus Culex Linnaeus

Linnaeus, 1758, Syst. Nat., 10: 602.

Type species: Culex pipiens Linnaeus.

22. Culex (Culex) epidesmus (Theobald)

Material examined: Two females, 3.ix.1997, Patiala, coll. J. Kaur.

23. Culex (Culex) gelidus Theobald

Material examined: One female, one male, 1.x.1997, two females, one male, 16.x.1998, three males, 19.xi.1999, Patiala, coll. J. Singh.

\section{Culex (Culex) whitmorei (Giles)}

Material examined: Two females, 5.vii.1997, Patiala; one female, 26.vii.1997, Mohie, Ludhiana, coll. J. Kaur.

\section{Culex (Culex) tritaeniorhynchus Giles}

Material examined: 52 females, five males, coll. J. Kaur, common species in Punjab from June to September.

\section{Culex (Culex) pseudovishnui Colless}

Material examined: 99 Females, 38 males, coll. J. Kaur, common species in Punjab from June to November.

\section{Culex (Culex) vishnui Theobald}

Material examined: 77 females, 24 males, coll. J. Kaur, common species in Punjab from June to September.

\section{Culex (Culex) quinquefasciatus Say}

Material examined: 438 females, 238 males, coll. J. Kaur, common species in Punjab from May to December.

\section{Culex (Culex) bitaeniorhynchus Giles}

Material examined: Six females, two males, 19.vii.1997, Mohie, Ludhiana; eight females, three males, 21.ix.1997, Patiala, coll. J. Kaur, J. Singh.

\section{Subgenus Lutzia Theobald}

Theobald, 1903, Monog. Culic., 3: 155.

Type species: Lutzia bigoti Theobald

\section{Culex (Lutzia) fuscanus Wiedemann}

Material examined: One male, 6.x.1997, one male, 21.xii.1997, three females, four males, 8.xi.1998, Patiala, coll. J. Kaur.

\section{REFERENCES}

Barraud, P.J. (1934). The Fauna of British India Diptera including Ceylon and Burma, 5, Taylor and Francis, London, 463pp.

Christophers, S.R. (1933). The Fauna of British India Diptera including Ceylon and Burma, 4, Taylor and Francis, London, 371pp.

Colless, D.H. (1956). Notes on the Culicine Mosquitoes of Singapore. II. The Culex vishnui group (Diptera, Culicidae), with descriptions of two new species. Ann. Trop. Med. Para., 51: 87-101.

Kirti, J.S. and J. Kaur (1999). Mosquitoes of Malwa region of Punjab (India). Geobios New Reports 18: 75-76.

Puri, I.M. (1954). Synoptic table for the identification of the Anopheline Mosquitoes of India. Health Bulletin 10: 1-57.

Sagandeep, V.C. Kapoor and J.S. Grewal (1994). Some mosquito (Diptera: Culicidae) species of Punjab and Himachal Pradesh. Journal of Insect Science 7: 48-50.

Wattal, B.L. and N.L. Kalra (1961). Regionvise pictorial keys to the female Indian Anopheles. Bulletin of National Society Indo Malayan Mosquito Diseases 9: 85-138.

\section{ACKnowledgements}

Authors are thankful to Director, NICD, New Delhi for providing relevent literature. 